\title{
Canine Wound Myiasis Caused by Lucilia sericata (Diptera: Calliphoridae) in Korea
}

\author{
Seongjun Choe', Dongmin Lee', Hansol Park', Hyeong-Kyu Jeon¹, Hakhyun Kim², Ji-Houn Kang², Cha-Ho Jee², \\ Keeseon S. Eom ${ }^{1, *}$ \\ ${ }^{1}$ Department of Parasitology, Medical Research Institute and Parasite Resource Bank, School of Medicine, Chungbuk National University, Cheongju \\ 28644, Korea; 'Laboratory of Veterinary Internal Medicine, Department of Veterinary Medicine, College of Veterinary Medicine, Chungbuk National \\ University, Cheongju 28644, Korea; ${ }^{3}$ Laboratory of Veterinary Parasitology, Department of Veterinary Medicine, College of Veterinary Medicine and \\ Research Institute of Veterinary Medicine, Chungbuk National University, Cheongju 28644, Korea
}

\begin{abstract}
Myiasis is a relatively common infection of animals kept as pets, although only 1 case of canine myiasis has been described so far in the Republic of Korea. In the present study, we report an additional case of canine wound myiasis with identification of its causative agent, Lucilia sericata. An 8-year-old male Siberian husky dog was referred with anorexia, vomiting, and diarrhea to the Chungbuk National University Veterinary Medical Center, Cheongju-si (city), Chungcheongbuk-do (province), Korea in July 2013. Physical examination indicated the patient had a deep wound filled with a maggot swarm as a left gluteal lesion. A total of 216 maggots were removed by forceps, and the wounded area was sponged with gauzes and disinfected with $70 \%$ alcohol and a povidone-iodine solution. After daily care and suturing the wound, the patient was discharged at day 19 after admission. Recovered worms possessed morphological characteristics similar to those of $L$. sericata, namely, a sub-cylindrical body with 6-8 lobed anterior spiracles, round shaped with a button surrounded by a peritremal ring with no gaps, and similar distances between dorsal, median, and outer papillae of the 12th segment. Additionally, cox1 partial sequences (528 bp) obtained in the present study showed 100\% identity with those of $L$. sericata (GenBank no. KT272854.1). L. sericata is indicated as a pathogen of myiasis infection not only in humans, but also in animals kept as pets in Korea.
\end{abstract}

Key words: Lucilia sericata, cox1, wound myiasis, identification

\section{INTRODUCTION}

Myiasis is an infestation by dipterous larvae [1]. Various species of dipterans are known as possible causes of infection in animals, such as livestock, wildlife, and even humans. Myiasisproducing dipterans can be divided into 3 groups: specific, semi-specific, and accidental myiasis-producing dipterans $[2,3]$. The myiasis caused by these 3 groups is known as obligatory, facultative, and accidental, respectively [4].

Facultative myiasis is caused by flies that normally choose breeding sites, such as dead bodies or vegetable matter, but occasionally develops in the tissues of living humans and animals. The female flies are attracted by the stinking discharge from inflammatory regions, wounded skin, or diseased organs,

- Received 27 June 2016, revised 23 July 2016, accepted 23 July 2016

*Corresponding author (kseom@chungbuk.ac.kr)

(c) 2016, Korean Society for Parasitology and Tropical Medicine

This is an Open Access article distributed under the terms of the Creative Commons Attribution Non-Commercial License (http://creativecommons.org/licenses/by-nc/4.0) which permits unrestricted non-commercial use, distribution, and reproduction in any

medium, provided the original work is properly cited. and lay or drop their eggs and larvae on these areas $[2,3]$.

Several cases of human myiasis involving facultative myiasis have been reported in Korea [5-13]. In the veterinary field, various cases were frequently mentioned between veterinarians comprising small-animal clinicians, but also wildlife veterinarians (personal communication); however, only 1 case has been currently reported, and it lacks species identification [14]. The purpose of the present study is to report a second case of canine myiasis in Korea. In addition, we provide morphological and molecular identification of the causative agent, Lucilia sericata.

\section{CASE DESCRIPTION}

A canine in the form of an 8-year-old male Siberian husky was referred with anorexia, vomiting, and diarrhea to the Chungbuk National University Veterinary Medical Center, Cheongju-si (city) in July 2013. Physical examination revealed a deep wound near the left hip. The wound was filled with maggots and pus-like fluid with an intense odor (Fig. 1). All 

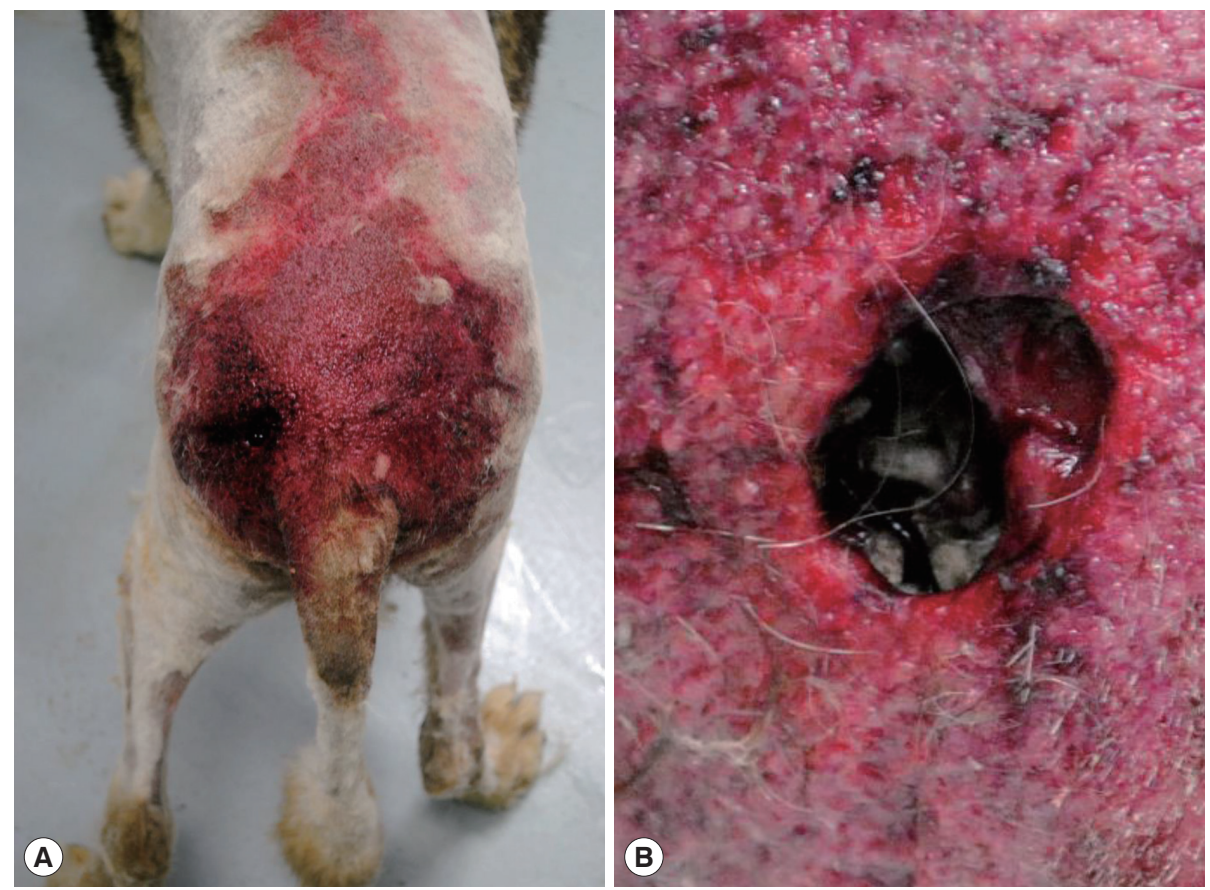

Fig. 1. Outlook of the case. (A) Overview of the wound. (B) Wound crowded with maggot swarm.

maggots were removed using forceps, and the affected area was sponged with gauzes and disinfected with $70 \%$ alcohol and a povidone-iodine solution every day. Due to the wide range of skin and tissue loss, wound closure was not available. We left the wound open until the region recovered sufficiently for suture. The patient recovered and was discharged at day 19 after admission.

\section{Identification of species}

A total of 216 maggots were recovered from the wound. They were preserved with $70 \%$ ethyl alcohol and transferred to a parasitology laboratory for identification. After measuring their body size, the fixed maggots $(\mathrm{n}=10)$ were skinned off via a longitudinal incision on the ventral side. For detailed morphological observations, the skins were immersed in $10 \%$ aqueous $\mathrm{KOH}$ for $24 \mathrm{hr}$ for maceration, mounted with polyvinyl alcohol, and then observed under a light microscope. Morphological identification of the maggots was based on a key to species provided by James [15].

The maggots were all third-instar larvae, with an average length of $11 \mathrm{~mm}$ and width of $2 \mathrm{~mm}$. The body was whitishbeige color, and internal contents were observed as somewhat red. The sub-cylindrical body was tapered slightly anteriorly and composed of 12 segments. The rear ends of each segment were covered with numerous tiny single-pointed spines that formed a spine band. The pseudocephalon had 2 antennae with a sensory papilla on each antenna, and 2 well-developed hooks were observed in the buccal cavity. A pigmented accessory oral sclerite was absent. Anterior spiracles were located at the anterolateral part of the body and were fan-shaped with 6-8 lobes. Posterior spiracles were located at the posterior end of the body, and were round shaped with a button, which was surrounded by a peritremal ring with no gap. Three straight slits were present on the posterior spiracle. The distances between the dorsal, median, and outer papillae of the 12th segment were almost similar (Fig. 2).

The internal contents of the maggots were used for molecular analysis. Genomic DNA was extracted from the internal contents using a PrimePrep Genomic DNA Extraction Kit from tissue (GeNet Bio, Daejeon, Korea). The genomic DNA was utilized for PCR using the protocols of Zhang et al. [16] with primer sets as follows: forward primer of 5'-CCTTTAGAATTGCAGTCTAATGTCA-3' and reverse primer of 5'-CCAAAGAATCAAAATAAATGTTG-3'. PCR products were sequenced using the sequencing machine, Applied Biosystems 3730xl DNA Analyzer (Cosmogenetech, Daejeon, Korea). Sequences were compared with those obtained from the GenBank database. We obtained 528 bp of cox1 partial sequences and all showed 100\% identity 

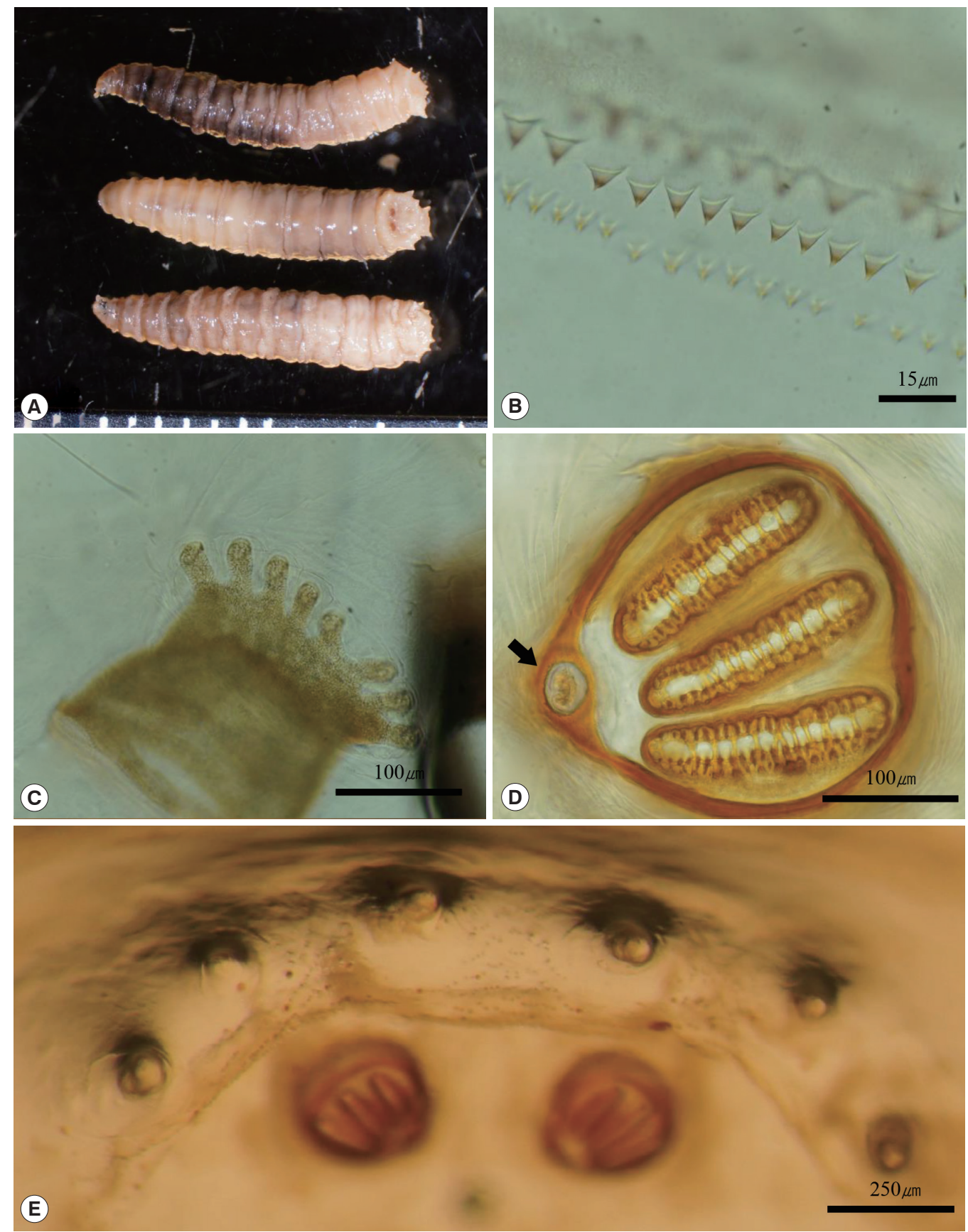

Fig. 2. Maggots recovered from wound. (A) Three views (lateral, dorsal, and ventral) of third instar larvae. (B) Single pointed spines. (C) Anterior spiracle with 8 lobes. (D) Posterior spiracle with button (black arrow). (E) Similar distances between outer papillae.

with those of L. sericata (GenBank no. KT272854.1).

\section{DISCUSSION}

Although a large number of suspected cases of myiasis are recognized in veterinary fields within Korea, the causative agents have not been determined, until the present study.
Commonly, veterinary clinicians in Korea have only focused on treating myiasis, and identification of the pathogen was usually undervalued and not performed (personal communication). However, identification of fly species is important because in some species myiasis can develop into malignant cases [17]. For example, in cases of screwworm infections, like Cochliomyia hominivorax and Chrysomya bezziana, untreated in- 
fections can cause septicemia, secondary infections, and even host death. Larvae of fly species literally eat host tissues alive and can invade the vital organs of hosts [18].

Identification of dipterans is usually performed with morphological observations. However, when they are in a developmental stage such as eggs, larvae, or pupae, morphological differences between dipteran species are subtle [19]. Although morphological characteristics of adult flies are needed for reliable identification, rearing maggots to the adult stage is timeconsuming, and sometimes identification is not possible because of maggots' death prior to maturation into an adult. Even if adult flies are successfully recovered, species-level identification is very difficult without taxonomic knowledge [20]. As a consequence, several human myiasis cases have been reported in Korea without detailed species identification, or with identification only to the genus level $[5,8,10,12,13]$. To solve this problem, forensic entomologists introduced DNA-based identification, a method that allows easy identification of dipterans even if specimens are immature or dead [20]. In the present study, maggots exhibited typical morphological characteristics described in identification keys [15], and molecular analysis revealed that our specimens had cox1 partial sequences identical with those of $L$. sericata found in the GenBank database. These results provided sufficient evidence to regard our specimens as L. sericata. Additionally, our skin-offed techniques could be useful when the number of specimens available for identification is either small or limited to only 1 individual [10].

L. sericata is a metallic green fly commonly called the greenbottle fly. This species is a well-known causative agent of fly strike in sheep of the British Isles, South Africa, and New Zealand [15], and is known to cause myiasis in cats, dogs, and humans $[21,22]$. In Korea, causative agents of human myiasis cases were mostly identified or suspected as $L$. sericata or belonging to the genus Lucilia, with the exception of a case involving Phormia sp. [5-13]. Considering these cases, canine myiasis formerly reported in Korea was also thought to be caused by this species [14].

In the present case, the canine patient seemed to be vulnerable to myiasis due to several factors. Firstly, the dog was raised outdoors with improper management. Animals raised outdoors are easily accessed by flies. In particular, when animals are raised in a restricted space by fences or a leash with poor hygiene, the site becomes more attractive to flies because it is easily contaminated by accumulation of feces, urine, uneaten food, or other excrements. The attracted flies also cause irrita- tion and scratching, and this could lead to the development of wounds and even infestation [21]. Secondly, the dog displayed a poor body condition, and showed several symptoms, including anorexia, vomiting, and diarrhea. In addition, the wound opened near the proximal region of the tail and was therefore difficult for the patient to reach. Due to the poor health conditions and location of the wounded site, the patient seemed incapable of cleaning itself. Finally, as previously described for cases of canine myiasis in Korea, this case also occurred in the summer season [14]. Flies display furious activity during the summer season, and it is therefore commonly regarded as the season of highest risk.

In summary, we describe a second case of canine myiasis in Korea. The pathogen was identified as L. sericata using both morphological and molecular identification methods. The species seems to be an important pathogen of myiasis not only for human medicine, but also for veterinary medicine in Korea. Myiasis in pets is known as a relatively common disease and is a preventable problem [21]. The prevention of myiasis involves proper management of hygiene and health, especially for animals that are raised outdoors during the summer season.

\section{ACKNOWLEDGMENTS}

Parasite materials used in this study were provided by the Parasite Resource Bank of Korea, National Research Resource Center (2012-0000037), the Republic of Korea.

\section{CONFLICT OF INTEREST}

We have no conflict of interest related to this study.

\section{REFERENCES}

1. Hope FW. On insects and their larvae occasionally found in the human body. Trans Entomol Soc London 1840; 2: 256-271.

2. Patton WS. Notes on the myiasis-producing Diptera of man and animals. Bull Entomol Res 1921; 12: 239-261.

3. Patton WS. Some notes on Indian Calliphorinae. VII. Additional cases of myiasis caused by the larvae of Chrysomyia bezziana Vill., together with some notes on the Diptera which cause myiasis in man and animal. Indian J Med Res 1922; 9: 654-682.

4. McGraw TA, Turiansky GW. Cutaneous myiasis. J Am Acad Dermatol 2008; 58: 907-926.

5. Chung PR, Jung Y, Kim KS, Cho SK, Jeong S, Ree HI. A human case of internal myiasis in Korea. Korean J Parasitol 1996; 34: 151-154. 
6. Cho JH, Kim HB, Cho CS, Huh S, Ree HI. An aural myiasis case in a 54-year-old male farmer in Korea. Korean J Parasitol 1999; 37: $51-53$

7. Joo CY, Kim JB. Nosocomial submandibular infections with dipterous fly larvae. Korean J Parasitol 2001; 39: 255-260.

8. Choi E, Lim D, Na M, Yang J, Lee Y, Lee W. A case of internal myiasis of the respiratory system associated with pneumonia. Tubercul Respir Dis 2002; 53: 650-655.

9. Kim JS, Seo PW, Kim JW, Go JH, Jang SC, Lee HJ, Seo M. A nasal myiasis in a 76-year-old female in Korea. Korean J Parasitol 2009; 47: 405-407.

10. Kim JS, Kim JW, Lee HJ, Lee IY, Oh SA, Seo M. Ophthalmomyiasis caused by a Phormia sp. (Diptera: Calliphoridae) larva in an enucleated patient. Korean J Parasitol 2011; 49: 173-175.

11. Jang M, Ryu SM, Kwon SC, Ha JO, Kim YH, Kim DH, Jung SM, Lee SI, Sohn WM, Cha HJ, Ock M. A case of oral myiasis caused by Lucilia sericata (Diptera: Calliphoridae) in Korea. Korean J Parasitol 2013; 51: 119-123.

12. Heo SJ, Lee MJ, Park CM, Kim JS. A case of nosocomial nasal myiasis in comatose patient. Korean J Otorhinolaryngol-Head Neck Surg 2013; 56: 664-666.

13. Han JU, Suk SH, Im JS, Kim BY. A case of endoscopic removal of nasal myiasis in cerebral infarction patient. J Rhinol 2015; 22: 51-54.

14. Choi J, Kim H, Na J, Kim SH, Park C. Cutaneous myiasis associ- ated with tick infestations in a dog. J Vet Clin 2015; 32: 473-475.

15. James MT. The flies that cause myiasis in man. Washington DC, USA. US Government Printing Office. 1947, p 175.

16. Zhang Y, Park SH, Yu DH, Yu GY, Jung HJ, Jo TH, Hwang JJ. Comparison of mitochondrial cytochrome c oxidase subunit I (CO I) sequences of five blow fly species in Korea. Korean J Legal Med 2006; 30: 154-159.

17. Singh TS, Rana D, Urogenital myiasis caused by Megaselia scalaris (Diptera: Phoridae): case report. J Med Entomol 1989; 26: 228229.

18. Catts EP, Mullen GR. 16. Myiasis (Muscoidea, Oestroidea). In Mullen GR, Durden LA eds, Medical and Veterinary Entomology. USA. Elsevier Science. 2002, pp 317-348.

19. Liu D, Greenberg B. Immature stages of some flies of forensic importance. Ann Entomol Soc Am 1989; 82: 80-93.

20. Sperling FA, Anderson GS, Hickey DA. A DNA-based approach to the identification of insect species used for postmortem interval estimation. J Forensic Sci 1994; 39: 418-427.

21. Anderson GS, Huitson NR. Myiasis in pet animals in British Columbia: The potential of forensic entomology for determining duration of possible neglect. Can Vet J 2004; 45: 993-998.

22. Pezzi M, Whitmore D, Chicca M, Lanfredi M, Leis M. Traumatic myiasis caused by an association of Sarcophaga tibialis (Diptera: Sarcophagidae) and Lucilia sericata (Diptera: Calliphoridae) in a domestic cat in Italy. Korean J Parasitol 2015; 53: 471-475. 
